Article

\title{
Iterative Signal Detection Scheme Using Multilayer Perceptron for a Bit-Patterned Media Recording System
}

\author{
Seongkwon Jeong ${ }^{(\mathbb{D})}$ and Jaejin Lee * \\ School of Electronic Engineering, Soongsil University, 369 Sangdo-ro, Dongjak-gu, Seoul 06978, Korea; \\ seongkwon@ssu.ac.kr \\ * Correspondence: zlee@ssu.ac.kr; Tel.: +82-2-820-0901
}

Received: 10 November 2020; Accepted: 5 December 2020; Published: 9 December 2020

\begin{abstract}
Because of the physical and engineering problems of conventional magnetic data storage systems, bit-patterned media recording (BPMR) is expected to be a promising technology for extending the storage density to beyond $1 \mathrm{~Tb} / \mathrm{in}^{2}$. To increase the storage density in BPMR systems, the separation between islands in both down- and cross-track directions must be reduced; this reduction results in two-dimensional interference from neighboring symbols in those directions, which is a major performance degradation factor in BPMR. Herein, we propose an iterative signal detection scheme between a Viterbi detector and a multilayer perceptron to improve the performance of a BPMR system. In the proposed signal detection scheme, we use the modified output of a multilayer perceptron as a priori information to improve equalization and extrinsic information to decrease the effect of intertrack interference.
\end{abstract}

Keywords: bit-patterned media recording; iterative decoding; multilayer perceptron; signal detection

\section{Introduction}

Owing to the increased demand for storage of digital data in the information age, conventional magnetic data storage systems have reached their storage density limit because of physical and engineering problems, such as the superparamagnetic limit. To satisfy the increasing demand, overcome the aforementioned limitation, and expand the storage density to $4 \mathrm{~Tb} / \mathrm{in}^{2}$, bit-patterned media recording (BPMR) has been recognized as one of the promising technologies [1]. In BPMR, each data bit is stored in a single-domain magnetic island that is distributed in both the down- and cross-track directions. Because nonmagnetic regions exist between single-domain magnetic islands, BPMR can reduce track edge and transition noise, nonlinear bit shift, and simplified tracking [2]. High storage density in BPMR can be achieved by reducing the distances between islands in both the down- and cross-track directions. However, this reduction results in major challenges of intersymbol interference (ISI) and intertrack interference (ITI) in the down- and cross-track directions, respectively; as such, two-dimensional (2D) ISI must be considered [3]. These error factors disrupt signal detection and degrade BPMR system performance. In addition, when the read head cannot continue at the center of the main data track, the system performance is degraded owing to track misregistration (TMR). To overcome performance degradation from these error factors, signal processing schemes such as signal detection, error control coding, and modulation coding schemes must be employed [4-7]. To mitigate ISI and ITI effects, a 2D equalizer and one-dimensional (1D) partial response (PR) target based on the minimum mean square error (MMSE) criterion was proposed for the BPMR system [4]. In recent years, signal processing techniques using multilayer perceptron (MLP), which is a class of feedforward artificial neural networks, have been employed to estimate and recover data sequences [8-10]. Herein, 
we propose an iterative signal detection scheme between the Viterbi detector and MLP. In the proposed detection scheme, to improve the BPMR system performance, the modified output of the MLP is used as a priori information to improve equalization and extrinsic information to cancel the effect of ITI. The rest of this paper is organized as follows: In Section 2, the BPMR channel model is briefly introduced; in Section 3, we explain the proposed iterative signal detection scheme using MLP; in Section 4, we discuss simulation and results; and in Section 5, the conclusions of this study are stated.

\section{Bit-Patterned Media Recording (BPMR) Channel Model}

To record user data into a medium, an analytical 2D Gaussian pulse response is used for BPMR channel modeling. For modeling simplicity, the 2D Gaussian pulse response without considering media noise and write errors is expressed as follows [11]:

$$
P(z, x)=A \cdot \exp \left\{-\frac{1}{2 c^{2}}\left[\left(\frac{z}{P W_{z}}\right)^{2}+\left(\frac{x}{P W_{x}}\right)^{2}\right]\right\}
$$

where $A$ is the normalized peak amplitude; $c$ represents the relationship between the PW50, which is a parameter of the pulse width at half of the peak amplitude and the standard deviation of the Gaussian function; $P W_{z}$ and $P W_{x}$ are the PW50 of the cross- and down-track pulses, respectively. In this study, we set $A=1, c=1 / 2.3548, P W_{z}=24.8 \mathrm{~nm}$, and $P W_{x}=19.4 \mathrm{~nm}$. The 2D channel response $h_{m, n}$ can be obtained by sampling the isolated pulse response as follows:

$$
h_{m, n}=P\left(m \cdot T_{z}+\Delta_{T M R}, n \cdot T_{x}\right)
$$

where $T_{z}$ and $T_{x}$ are the track pitch and bit period, respectively; and $\Delta_{T M R}$, which is expressed as $\Delta_{T M R}=\left(T M R_{z} \times T_{z}\right) / 100$, is the read head offset. In this study, we set $T_{z}=T_{x}=14.5 \mathrm{~nm}$ to achieve an areal density of $3 \mathrm{~Tb} / \mathrm{in}^{2}$. The discrete BPMR readback signal, which is obtained by sampling $T_{z}$ and $T_{x}$, can be written as follows:

$$
r_{j, k}=\sum_{m=-M_{c}}^{M_{c}} \sum_{n=-N_{c}}^{N_{c}} a_{j-m, k-n} \cdot h_{m, n}+n_{j, k}
$$

where $M_{c}$ and $N_{c}$ are the lengths of the interference from neighboring islands in the cross- and down-track directions, respectively; $a_{j, k} \in\{-1,1\}$ is the $k$-th recorded user data bit of the $j$-th track, and $n_{j, k}$ is the electronic noise modeled as additive white Gaussian noise with zero mean and variance $\sigma^{2}$. In this study, we set $M_{c}=N_{c}=1$ for simplicity.

\section{Proposed Iterative Signal Detection Scheme}

A block diagram of the proposed iterative detection scheme is shown in Figure 1. The partial response maximum likelihood (PRML) detector, which comprises a PR equalizer and a maximum likelihood sequence detector, is used to manage the ISI and ITI. In this study, a 2D equalizer and a 1D PR target based on the MMSE criterion and Viterbi detectors is used for equalization and detection, respectively.

An MLP is utilized to improve the system performance using the MLP output as the a priori information for equalization and the extrinsic information for detection. Figure 2 shows the structure of the MLP that is composed of three types of layers, i.e., input layer, hidden layer, and output layer. In all neurons in the hidden layers in the MLP, a rectified linear unit $(\operatorname{ReLU})$, expressed as $f(y)=\max (0, y)$ is used as the activation function. For the neuron in the output layer of the MLP, we use a sigmoid function, $s(v)=1 /\left(1+e^{-v}\right)$ as the activation function. For training, the MLP is trained by Keras in Tensorflow 2.0, which is a high-level neural network application programming interface, written in Python and capable of operating on top of TensorFlow [12]. To obtain the weights that minimize the loss function, adaptive moment estimation (Adam) is used as the optimizer [13]. After user data are passed through BPMR channel, the proposed detection scheme is performed as follows: (Step 1) First, 
the readback signal $r_{j, k}=\left[r_{j+M_{m}, k+N_{m}} r_{j+M_{m}, k+N_{m}-1} \cdots r_{j, k} \cdots r_{j-M_{m}, k-\left(N_{m}-1\right)} r_{j-M_{m}, k-N_{m}}\right]$ is used as the input sequence of the MLP, and then the MLP is implemented. (Step 2) To exploit the MLP output $s(v)$ as reliable information, the information $p_{j, k}$, which is used as a priori information in the equalizer and extrinsic information in the detector, is modified as follows:

$$
p_{j, k}=\alpha \cdot(2 \cdot s(v)-1)
$$

where $\alpha$ is the scaling factor of the information. (3) Using $\left(2 M_{w}+1\right) \times\left(2 N_{w}+1\right) 2 D$ equalizer with a $\left(2 N_{g}+1\right) 1 D$ PR target, the equalizer output $s_{j, k}$ and desired output $d_{j, k}$ can be calculated as follows:

$$
\begin{gathered}
s_{j, k}=\sum_{m=-M_{w}}^{M_{w}} \sum_{n=-N_{w}}^{N_{w}} w_{m, n} \cdot\left(r_{j-m, k-n}+p_{j-m, k-n}\right)=\sum_{m=-M_{w}}^{M_{w}} \sum_{n=-N_{w}}^{N_{w}} w_{m, n} \cdot q_{j-m, k-n}=\mathbf{w}^{T} \mathbf{q}_{j, k} \\
d_{j, k}=\sum_{n=-N_{g}}^{N_{g}} g_{n} \cdot a_{j, k-n}=\mathbf{g}^{T} \mathbf{a}_{j, k}
\end{gathered}
$$

where $\mathbf{w}^{T}=\left[w_{-M_{w},-N_{w}} w_{-M_{w},-N_{w}+1} \cdots w_{0,0} \cdots w_{M_{w}, N_{w}-1} w_{M_{w}, N_{w}}\right]$ is the equalizer coefficient, $\mathbf{q}_{j, k}=$ $\left[q_{j+M_{w}, k+N_{w}} q_{j+M_{w}, k+N_{w}-1} \cdots q_{j, k} \cdots q_{j-M_{w}, k-\left(N_{w}-1\right)} q_{j-M_{w}, k-N_{w}}\right]$ is the equalizer input that combines the readback signal $r_{j, k}$ and the a priori information $p_{j, k}, \mathbf{g}^{T}=\left[\begin{array}{lll}g_{-N_{g}} & \cdots & g_{N_{g}}\end{array}\right]$ is the target polynomial coefficients, and $\mathbf{a}_{j, k}=\left[\begin{array}{lll}a_{k+N_{g}} & \cdots & a_{k-N_{g}}\end{array}\right]$ is the user data sequences. To design the equalizer and target, we utilize the MMSE criterion $[14,15]$. The mean square error can be computed as

$$
E\left[e_{j, k}^{2}\right]=\mathbf{w}^{T} \mathbf{Q} \mathbf{w}-2 \mathbf{w}^{\mathbf{T}} \mathbf{C} \mathbf{g}+\mathbf{g}^{\mathbf{T}} \mathbf{A} \mathbf{g}-2 \lambda\left(\mathbf{J}^{\mathbf{T}} \mathbf{g}-1\right)
$$

where $\mathbf{Q}=E\left[\mathbf{q}_{j, k} \mathbf{q}_{j, k}^{T}\right]$ is the auto-correlation matrix of size $\left(2 M_{w}+1\right)\left(2 N_{w}+1\right)$ by $\left(2 M_{w}+1\right)\left(2 N_{w}+1\right)$, $\mathbf{C}=E\left[\mathbf{q}_{j, k} \mathbf{a}_{j, k}^{T}\right]$ is the cross-correlation matrix of size $\left(2 M_{w}+1\right)\left(2 N_{w}+1\right)$ by $\left(2 N_{g}+1\right), \mathbf{A}=E\left[\mathbf{a}_{j, k} \mathbf{a}_{j, k}^{T}\right]$ is the auto-correlation matrix of size $\left(2 N_{g}+1\right)$ by $\left(2 N_{g}+1\right), \lambda$ is the Lagrange multiplier, and $\mathbf{J}$ is the vector of length $\left(2 N_{g}+1\right)$ such that the second element is 1 and the remaining elements are 0 . By taking the derivatives of the righthand side of Equation (7) with respect to $\lambda$, $\mathbf{g}$, and $\mathbf{w}$, respectively, and setting the resulting expressions to zero, the optimized target and equalizer coefficients can be obtained as follows:

$$
\begin{gathered}
\lambda=\frac{1}{\mathbf{J}^{T}\left(\mathbf{A}-\mathbf{C}^{T} \mathbf{Q}^{-1} \mathbf{C}\right)^{-1} \mathbf{J}} \\
\mathbf{g}=\lambda\left(\mathbf{A}-\mathbf{C}^{T} \mathbf{Q}^{-1} \mathbf{C}\right)^{-1} \mathbf{J} \\
\mathbf{w}=\mathbf{Q}^{-1} \mathbf{C g}
\end{gathered}
$$

(Step 4) To consider the effect of ITI from the adjacent track, the branch metric in the Viterbi detector after equalization is calculated as follows:

$$
\beta_{j, k}\left(t_{i}, t_{i+1}\right)=\left\{s_{j, k}-\left(g_{N_{g}} \cdot a_{j, k+N_{g}}\left(t_{i+1}\right)+\cdots+g_{-N_{g}} \cdot a_{j, k-N_{g}}\left(t_{i}\right)-\left(p_{j-1, k}+p_{j+1, k}\right)\right\}^{2}\right.
$$

where $t_{i}$ is the state; $a\left(t_{i}\right)$ is the decision at a state; and $p_{j-1, k}$ and $p_{j+1, k}$ are extrinsic information from the upper and lower tracks, respectively. (Step 5 ) To deliver appropriate information as a priori information for the MLP, the Viterbi output $\hat{a}_{j, k}$ is modified to the following:

$$
\tilde{a}_{j, k}=\alpha \cdot \hat{a}_{j, k} .
$$


In addition, the combined value of the modified output of the Viterbi detector $\widetilde{a}_{j, k}$ and the readback signal is input to the MLP. (Step 6) During the iterative signal detection, the MLP delivers the output only to the Viterbi detector, and the Viterbi detector delivers the output only to the MLP. In addition, the Viterbi detector outputs the estimated data. When the number of iterations is zero, Steps 1 to 5 are performed.

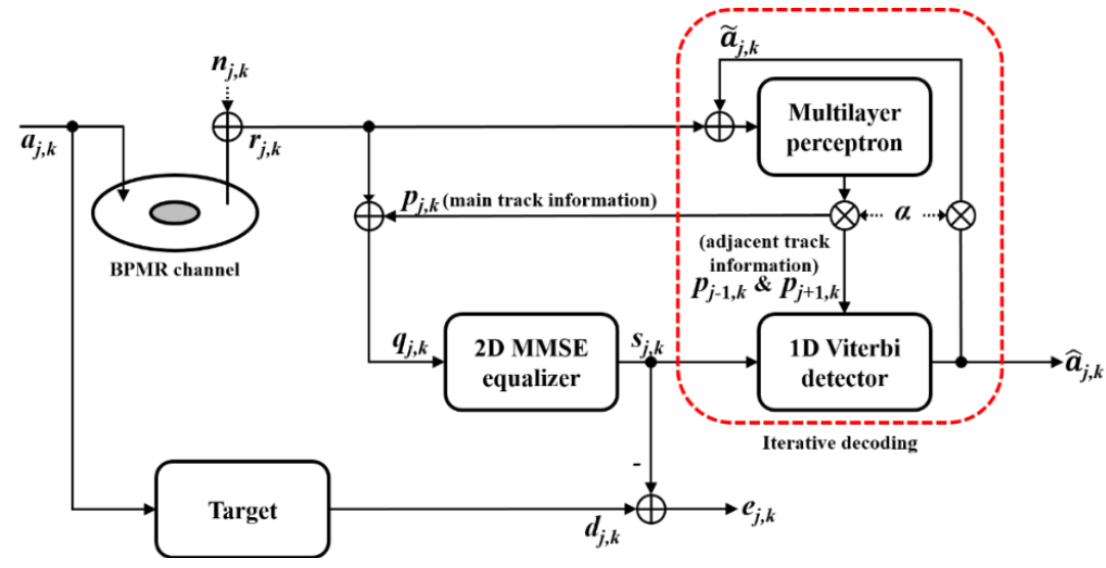

Figure 1. Block diagram of proposed bit-patterned media recording (BPMR) system model.

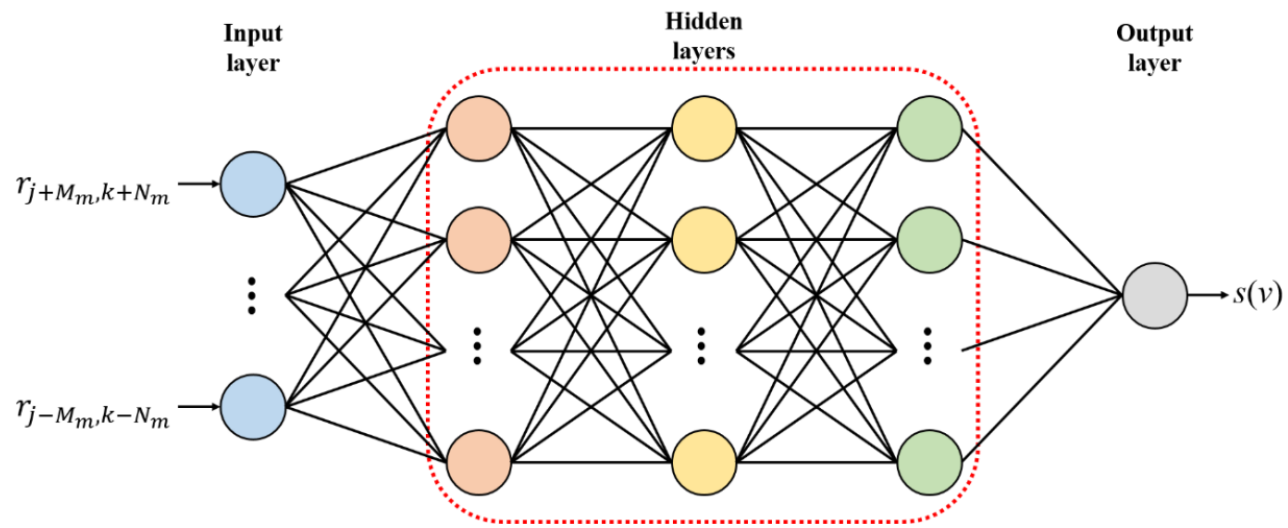

Figure 2. Multilayer perceptron (MLP) structure.

\section{Simulation and Results}

In this simulation, we set $M_{m}=N_{m}=1$ for the MLP input sequences, $M_{w}=1, M_{m}=5$ for the 2D equalizer of width 3 and length 11 , and $N_{g}=1$ for the PR target of length 3 . The signal-to-noise ratio (SNR) is defined as $10 \log _{10}\left(1 / \sigma^{2}\right)$. At each SNR, 810,000 bits for training the MLP and 2,250,000 bits for simulating the proposed detector are used. Figure 3 shows the bit error rate (BER) performance depending on the scaling factor $\alpha$ when SNR $=16 \mathrm{~dB}$, and one hidden layer with 16 neurons in the MLP is selected. Because the proposed detector demonstrates the minimum BER performance when $\alpha$ is 0.1 , we set $\alpha$ to 0.1 as the scaling factor.

Figure 4 presents the BER performance comparisons among the conventional PRML detector, MLP alone, and the proposed detector according to the SNR at a TMR of $\%$. In the conventional PRML, the readback signal is detected without the MLP and iteration. In the MLP scheme alone, the readback signal is estimated only by the MLP. As shown in Figure 3, at BER $=10^{-4}$, the proposed detector with one hidden layer with 16 neurons without iteration provides an SNR gain of approximately $2 \mathrm{~dB}$ and $1 \mathrm{~dB}$ as compared with the conventional PRML detector and MLP alone, respectively. Subsequently, to compare the BER performance based on the number of hidden layers and neurons in the MLP, we apply two types of MLP to the proposed detector. One is a hidden layer with 16 neurons (Case 1) 
and the other is three hidden layers with 128 neurons in each layer (Case 2). Without iteration, the BER performance of Case 2 improves by $1 \mathrm{~dB}$ as compared with that of Case 1. Finally, to compare the BER performance with respect to the number of iterations, we simulate the proposed detector iterating numbers from 0 to 2. Even when the number of iterations increases at the BER of $10^{-4}$, the performance of Case 1 without iteration is similar to that of Case 1 with one and two iterations. However, when the BER is $10^{-5}$, the BER of Case 2 with two iterations is higher by approximately 0.3 and $0.1 \mathrm{~dB}$ as compared with Case 2 without iteration and with one iteration, respectively.

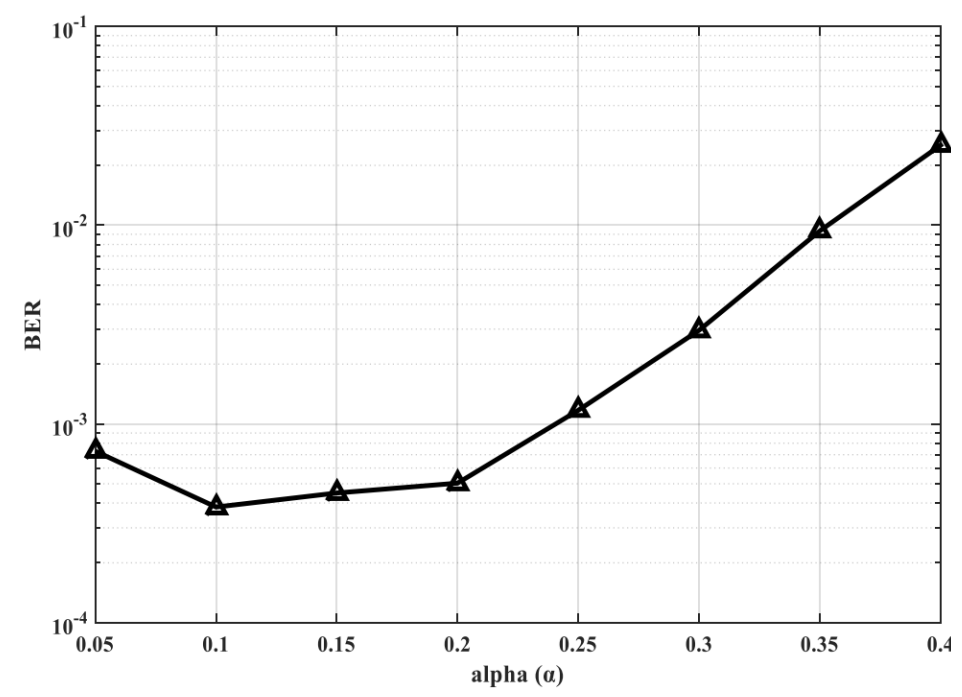

Figure 3. Bit error rate (BER) performance of proposed signal detection scheme based on scaling factor $\alpha$.

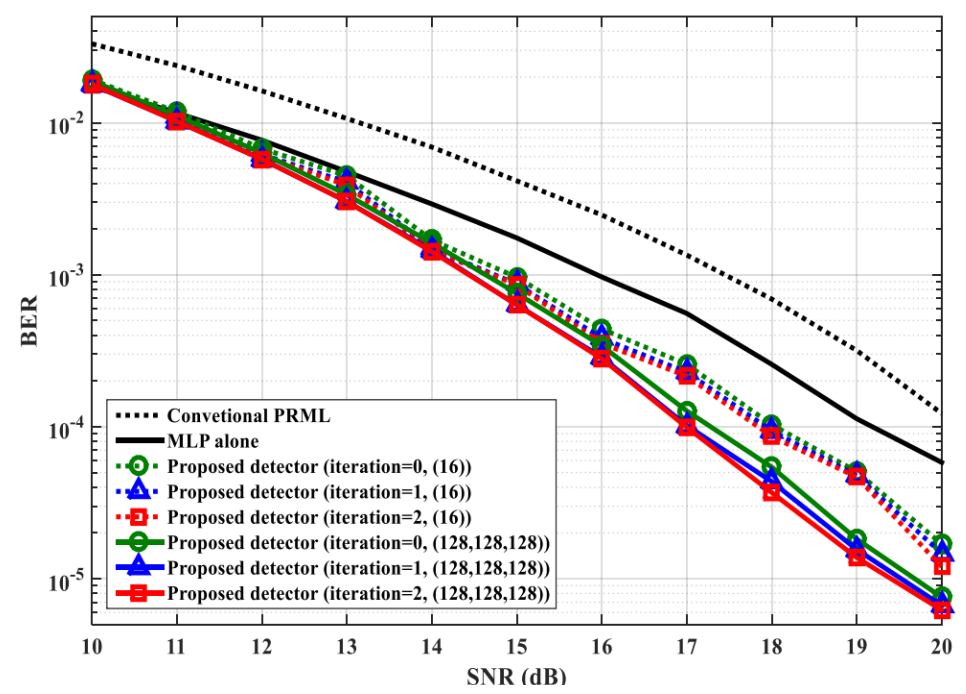

Figure 4. BER performance comparisons among conventional partial response maximum likelihood (PRML) detector, MLP alone, and proposed detector based on signal-to-noise ratio (SNR) at track misregistration (TMR) of $0 \%$.

Figure 5 illustrates the BER performance at SNR of $20 \mathrm{~dB}$ when the TMR is varied from $0 \%$ to $30 \%$. The proposed detector (Cases 1 and 2) indicates better BER performance as compared with the conventional PRML and MLP alone. Moreover, increasing the number of hidden layers, neurons, and iterations improves the BER performance. 


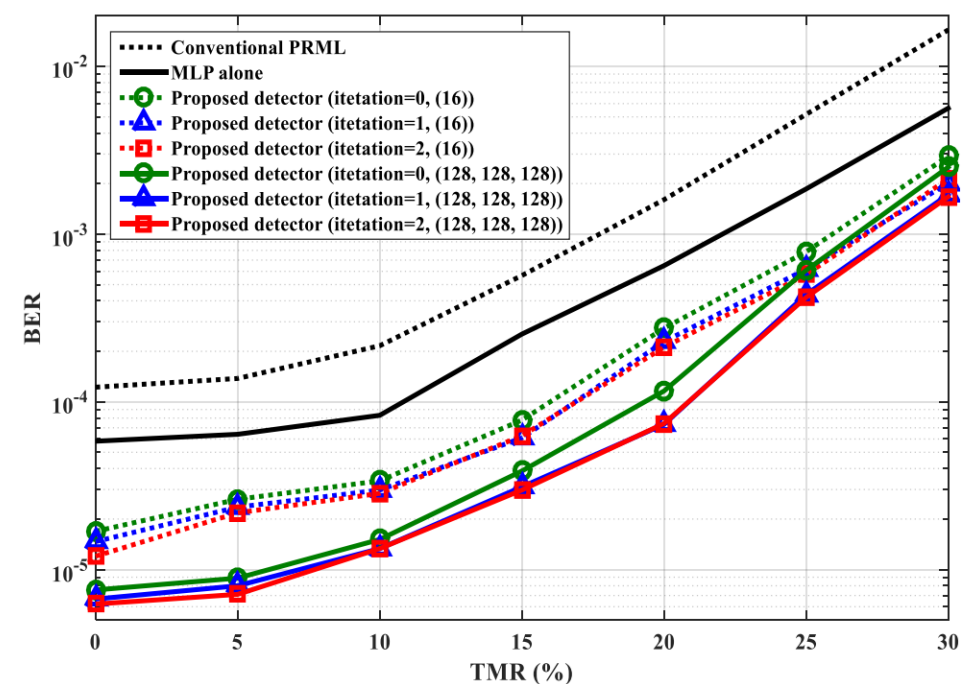

Figure 5. BER performance depending on TMR at SNR of $20 \mathrm{~dB}$.

When the proposed detector is used, the response time is increased as compared with a conventional PRML detector. However, since the proposed detector can cancel the ITI effect from neighboring islands, it shows better BER performance than a conventional PRML and MLP alone. Hence, to use the proposed detector, the trade-off between complexity (or cost) and BER performance should be considered.

\section{Conclusions}

Herein, we propose an iterative detection scheme between Viterbi detection and MLP to improve the performance of the BPMR system. Because the scaled output of the MLP is used as a priori information to improve equalization and extrinsic information to cancel the effect of ITI, the proposed detector provides improved BER performance as compared with a conventional PRML and MLP alone. In addition, we investigate the performance based on the number of hidden layers, neurons, and iterations. Although the computational complexity of Case 2 is higher than that of Case 1, the results indicate a better BER performance for Case 2. Therefore, depending on the circumstances, the tradeoff between computational complexity and performance should be considered.

Author Contributions: S.J. contributed to this work in experiment planning, experiment measurements, data analysis and manuscript preparation; J.L. contributed to experiment planning, data analysis, and manuscript preparation. Both authors have read and agreed to the published version of the manuscript.

Funding: This research was supported by Basic Science Research Program through the National Research Foundation of Korea(NRF) funded by the Ministry of Science and ICT(NRF-2017R1A5A1015596).

\section{References}

1. Richter, H.J.; Dobin, A.Y.; Heinonen, O.; Gao, K.Z.; Veerdonk, R.J.M.V.D.; Lynch, R.T.; Xue, J.; Weller, D.; Asselin, P.; Erden, M.F.; et al. Recording on bit-patterned media at densities of $1 \mathrm{~Tb} / \mathrm{in}^{2}$ and beyond. IEEE Trans. Magn. 2006, 42, 2255-2260. [CrossRef]

2. White, R.L.; New, R.M.H.; Pease, R.F.W. Patterned media: A viable route to $50 \mathrm{Gbit} / \mathrm{in}^{2}$ and up for magnetic recording. IEEE Trans. Magn. 1997, 33, 990-995. [CrossRef]

3. Nabavi, S.; Kumar, B.V.K.V.; Bain, J.A. Two-dimensional pulse response and media noise modeling for bit-patterned media. IEEE Trans. Magn. 2008, 44, 3789-3792. [CrossRef]

4. Ng, Y.; Cai, K.; Kumar, B.V.K.V.; Zhang, S.; Chong, T.C. Modeling and two-dimensional equalization for bit-patterned media channels with media noise. IEEE Trans. Magn. 2009, 45, 3535-3538.

5. Nguyen, T.A.; Lee, J. Effective generalized partial response target and serial detector for two-dimensional bit-patterned media recording channel including track mis-registration. Appl. Sci. 2020, 10, 5738. [CrossRef]

6. Jeong, S.; Lee, J. Iterative LDPC-LDPC product code for bit patterned media. IEEE Trans. Magn. 2017, 53, 3100704. [CrossRef] 
7. Jeong, S.; Lee, J. Modulation code for reducing intertrack interference on staggered bit-patterned media recording. Appl. Sci. 2020, 10, 5295. [CrossRef]

8. Nair, S.K.; Moon, J. Data storage channel equalization using neural networks. IEEE Trans. Neural Netw. 1995, 8, 1037. [CrossRef] [PubMed]

9. Han, S.; Kong, G.; Choi, S. A detection scheme with TMR estimation based on multi-layer perceptrons for bit patterned media recording. IEEE Trans. Magn. 2019, 55, 3100704. [CrossRef]

10. Jeong, S.; Lee, J. Modulation code and multilayer perceptron decoding for bit-patterned media recording. IEEE Magn. Lett. 2020, 11, 6502705. [CrossRef]

11. Nabavi, S.; Kumar, B.V.K.V.; Bain, J.A.; Hogg, C.; Majetich, S.A. Application of image processing to characterize patterning noise in self-assembled nano-masks for bit-patterned media. IEEE Trans. Magn. 2009, 45, 3523-3526. [CrossRef]

12. Chollet, F. Deep Learning with Python, 1st ed.; Manning Publications: Shelter Island, NY, USA, 2018; pp. 61-62.

13. Kingma, D.P.; Ba, J. Adam: A method for stochastic optimization. In Proceedings of the International Conference Learn. Represent. (ICLR), San Diego, CA, USA, 5-8 May 2015.

14. Moon, J.; Zeng, W. Equalization for maximum likelihood detectors. IEEE Trans. Magn. 1995, 31, $1083-1088$. [CrossRef]

15. Kovintavewat, P.; Ozgunes, I.; Kurtas, E.; Barry, J.R.; McLaughlin, S.W. Generalized partial-response targets for perpendicular recording with jitter noise. IEEE Trans. Magn. 2002, 38, 2340-2342. [CrossRef]

Publisher's Note: MDPI stays neutral with regard to jurisdictional claims in published maps and institutional affiliations.

(C) 2020 by the authors. Licensee MDPI, Basel, Switzerland. This article is an open access article distributed under the terms and conditions of the Creative Commons Attribution (CC BY) license (http://creativecommons.org/licenses/by/4.0/). 